\title{
The Correction Algorithm of Coalition's Allocation with Random Payoffs
}

\author{
Zhongfu Qin ${ }^{a}$, Hanfei $\mathrm{Li}^{\mathrm{b}}$ and Xianrong Wei ${ }^{\mathrm{c}}$
}

\author{
College of Civil Engineering and Architecture, Zhejiang University, Hangzhou 310058, China \\ aqinzhongfu@zju.edu.cn, ${ }^{b}$ li_hanfei@zju.edu.cn, cweixianrr@163.com
}

Keywords: Coalition; Shapley; the Marginal Contribution; Risk Sharing.

\begin{abstract}
Combined with the characteristic of random payoffs for a coalition, this study mainly analyzes the achievement of profit allocation among the players with certain payoffs and random payoffs in coalition and study the inadequate of existing results. Then allowing for the effect of risk on the allocation of random payoffs among the players, this paper constructs a model about the risk sharing which is used to correct the allocation of coalition's random payoffs based on the Shapley value. The final result is a comprehensive reflection of the marginal contribution and the principle of risk compensation, which is quite reasonable.
\end{abstract}

\section{Introduction}

As the degree of market competition increased constantly and the macro-micro control policies of the government introduced gradually, more and more companies take the form of a strategic coalition in order to achieve leapfrogging development and a "win-win" for the players in coalition. So the assignment of payoffs becomes a tough problem during the contract signing due to the differences of the investment, resources, contributions, and the type or proportion of risk during the process of a project among the players. So how to design a scientific and rational profit allocation becomes a hot issue of concern in both industry and academia.

\section{The Allocation with Certain Payoffs}

At present, profit allocation to the players in coalition in our country increasingly departures from the way that the payoffs are allocated by contribution to the coalition, showing the situation uneven development: the profit allocation to players in coalition is just on the basis of different ratio of the initial investment ${ }^{[1]}$. The reason is that the initial investment is only a factor which makes a difference on the payoff of coalition and the decisive factors should be research, production, marketing and so on. the L. Liu and H. J. Tang etc ${ }^{[2]}$ use the theory of Shapley value to solve the problem from the perspective of each player's contribution to the coalition, which can get rid of the disadvantage of Nash-Harsanyi negotiation model and avoid sharing alike interest of Agile Vitual Enterprise. Nonetheless, owing to the risk sharing among the players is different to quantify, the marginal contribution ignores the different proportion of risk sharing and supposes that each player bears the same proportion of risk when solving the profit allocation. In other words, all the players' risk sharing is $1 / n$. It's impossible in real life. The reason is that even though the comments of each player on the risk sharing are identical, the proportion of risk sharing is not the ideal average proportion, $1 / n$, as a result of the effect of the aspiration, preference and ability on the risk sharing. Moreover, it's more common that the comments of each player are inconsistent.

J. H. Dai and H. X. Xue ${ }^{[3]}$ analyze the pros and cons of the Shapley value to solve the problem of profit allocation. And considering the impact of risk on the profit allocation, they propose a correction algorithm allowing for the risk, which is more reasonable. H. H. Zhou and H. L. Bao ${ }^{[4]}$ consider $^{\circ}$ business and knowledge spillover effect and revises the Shapley value through weighting computation, which is especially applicable for the allocation of profit in technological innovation alliance. Furthermore, Y. F. Shi and J. H. Gao ${ }^{[5]}$ investigate the satisfaction into the category of the profit allocation, and put forward the correction algorithm of the Shapley value by considering the comprehensive factors of investment, risk and satisfaction. The improvement model reflects better the profit pursuit of players in coalition and contributes to the stable and efficient. The solution above 
all derived from the theory of the Shapley value is under the assumption that the profit is definitely known by all the players in coalition, that is, the players exactly know the profit generated by different cooperation strategies and their own profit allocation in a particular coalition before cooperation. However, in many real-life situations the payoffs of the coalition to players are random and the players have to reach an agreement about the allocation before cooperation, which leads to restrict the application of the theory above.

\section{The Allocation with Random Payoffs}

To solve the problem of uncertainty payoffs on the coalition, many scholars introduce fuzzy theory into the Shapley value. The function of fuzzy Shapley, which Mares ${ }^{[6]}$ constructs on the basis classic theory of Shapley value ${ }^{[7]}$, is difficult to meet the three axioms - effectiveness, symmetry and additive; With the fuzzy mathematics theory and some other related theories, W. Chen ${ }^{[8]}$ proposes the interval fuzzy Shapley value, which meets the relative effectiveness, symmetry, additive, but it's not necessarily the solution to meet the conditions; $\mathrm{X}$. $\mathrm{H}$. $\mathrm{Yu}^{[9]}$ extends the three axioms of Shapley Value in the classical sense and gives a unique Shapley value fulfilled the three axioms. However, the solution is only applies to the payoff with interval function and can't solve the random payoff with other forms of payoff function.

With respect to the preference on random payoffs for the player i, J. M. Wang and H. J. Du ${ }^{[10]}$, with Timmer et al's ${ }^{[11]}$ assumptions, give the preference relations called "the preference with mathematical expectation". That is, the player $i$ would weekly prefer $\mathrm{X}$ to $\mathrm{Y}$ if and only if $E(X) \geq E(Y)$ for any payoffs $X$ and $Y$, where $E(X)$ is the mathematical expectation of $X$. However, as the future payoff is a random variable, the coalition's ultimate payoff is not necessarily equal to the mathematical expectation, which ignores that the player $i$ may consider the risk of the coalition and his own judgment at the same time to make sure the final value of the random payoffs. For example, the player $i$ can choose $j$ or $k$ to make up a coalition. If he cooperates with $j$, the random payoff $\mathrm{X}$ follows Gaussian distributionX $\sim N(3,2)$. And if he cooperates with $k$, the random payoff $Y$ follows Gaussian distribution $\mathrm{Y} \sim N(3,4)$. The player $i$ has no different preference on $\mathrm{X}$ and $\mathrm{Y}$ according to the result of mathematical expectation. But the player $i$ may actually prefer $\mathrm{X}$ to $\mathrm{Y}$ when considering the risk of random payoff.

Suijs et al ${ }^{[12]}$ introduce the random payoff into cooperative game theory to make it applicable to many other forms of payoff function, broadening its application scope greatly. In addition, they propose the player's subjective judgment to make up the shortage above. However, it does not take into account the effect of different risk sharing on the profit allocation, ignoring the principle of risk compensation, which is needed to further improve. This paper mainly gives a correction algorithm of the cooperative games with random payoff, so it emphasizes on the risk sharing among the players in coalition.

\section{The Correction Algorithm of Marginal Contribution Based on Risk Sharing}

During the process of the risk sharing before a project starts, suppose that the player $i$ 's scheme of risk sharing is $R_{i}=\left(r_{i 1}, r_{i 2}, \ldots, r_{i n}\right), 0 \leq r_{i j} \leq 1, \sum_{j=1}^{n} r_{i j}=1, r_{i j}$ is the coefficient of risk sharing that player $j$ should take in player $i$ 's mind. So the $n$ players give $n$ kinds of risk sharing schemes and it can make up a coefficient matrix of risk sharing:

$$
R=\left[\begin{array}{cccc}
r_{11} & r_{12} & \ldots & r_{1 n} \\
r_{21} & r_{22} & \ldots & r_{2 n} \\
\ldots & \ldots & \ldots & \ldots \\
r_{n 1} & r_{n 2} & \ldots & r_{n n}
\end{array}\right]
$$

Based on the improved optimization of group weight model ${ }^{[13]}$, this paper tries to make the coefficient matrix of risk sharing to be an actual risk sharing scheme $R^{*}=\left(r_{1}, r_{2}, \ldots, r_{n}\right)$ such that $\sum_{i=1}^{n} r_{i}=1$. The improved optimization of group weight model will be applied to the risk sharing 
such that the sum of squared difference between the actual risk sharing scheme $R^{*}$ and the risk sharing each player gives is lowest, or:

$$
\begin{aligned}
& \min f(r)=\omega_{1} \sum_{i=1}^{n}\left(r_{i}-r_{1 i}\right)^{2}+\omega_{2} \sum_{i=1}^{n}\left(r_{i}-r_{2 i}\right)^{2}+\cdots+\omega_{n} \sum_{i=1}^{n}\left(r_{i}-r_{n i}\right)^{2} \\
& \text { s.t } \sum_{i=1}^{n} r_{i}=1,0 \leq r_{i} \leq 1
\end{aligned}
$$

In Eq. (2), $\omega_{i}$ is the importance of player $i$ in alliance and $\sum_{i=1}^{n} \omega_{i}=1$. This paper holds that the more contribution a player can make to the payoff of the alliance, the larger power his words. So it's reasonable to make sure the importance of each player according to the marginal contribution, and it will be easy to be accepted by all the players. The core thought of Shapley theory is that the allocation on the random payoffs of player $i$ is based on the average value of every coalition which the player $i$ is $\mathrm{in}^{[14]}$. In this paper, assume that the player $i$ 's marginal contribution is $\phi_{i}$ on the basis of the model in Suijs et al and it is obviously that $\omega_{i}=\phi_{i}$. By solving the Eq. (2), the proportion of risk sharing to player $i$ is:

$$
r_{i}=\omega_{1} r_{1 i}+\omega_{2} r_{2 i}+\cdots+\omega_{n} r_{n i}=\sum_{j=1}^{n} \omega_{j} r_{j i}=\sum_{j=1}^{n} \phi_{j} r_{j i}
$$

By studying the risk sharing proportion of all players, we get the difference between the actual risk sharing proportion that player $i$ take and the average risk sharing proportion, $1 / n$. Denote by $\Delta R_{i}$ be the difference where $\Delta R_{i}=r_{i}-1 / n$ and $\sum_{i=1}^{n} \Delta R_{i}=0$.

Based on the marginal contribution and the principle of risk compensation, this paper defines the improved model of allocation on random payoffs:

$$
\begin{aligned}
R_{i} & =\phi_{i}+\Delta R_{i} \cdot R(N) \\
& =\phi_{i}+\left(r_{i}-1 / n\right) R(N) \\
& =\phi_{i}+\left(\sum_{j=1}^{n} \phi_{j} r_{j i}-1 / n\right) R(N)
\end{aligned}
$$

Where $\phi_{i}$ is the player i's marginal contribution and $R(N)$ is the random payoff of coalition $N$.

If $\sum_{j=1}^{n} \phi_{j} r_{j i}>1 / n$, it means the actual risk sharing proportion that player $i$ take is larger than the average proportion of risk sharing. So the player $i$ should get more risk compensation and the payoff adds $\left.\mid \sum_{j=1}^{n} \phi_{j} r_{j i}-1 / n\right) R(N) \mid$. Similarly, if $\sum_{j=1}^{n} \phi_{j} r_{j i}<1 / n$, player $i$ should get less risk compensation and the payoff reduces $\left.\mid \sum_{j=1}^{n} \phi_{j} r_{j i}-1 / n\right) R(N) \mid$.

Example: Suppose that the player 1, 2, 3 make up a coalition. The marginal contributions based on the Shapley value with random payoff are $\phi_{1}=0.4 ; \phi_{2}=0.3 ; \phi_{3}=0.3$ and the random payoff of coalition is $R(N)$. Concerning the scheme of risk sharing among the players is:

$$
R=\left[\begin{array}{lll}
0.4 & 0.4 & 0.2 \\
0.3 & 0.3 & 0.4 \\
0.3 & 0.2 & 0.5
\end{array}\right]
$$

Then according to Eq. (3), it's easy to get the actual risk sharing for each player: $r_{1}=0.34$; $r_{2}=0.31 ; r_{3}=0.35$.

So the final profit allocations are $R_{1}=0.4+(0.34-1 / 3)=0.407 ; R_{2}=0.277$ and $R_{3}=$ 0.316 . It is easy understood that correction algorithm of coalition's random payoffs is a comprehensive result of the marginal contribution and the principle of risk compensation.

\section{Conclusion}

This paper studies the problem of optimal allocation of random payoffs to all the players in coalition. It proposes the improved model of allocation on random payoffs by concerning the marginal contribution and the principle of risk compensation, which is quite reasonable. However, this article just gives a rough scheme of risk sharing. It's needed to have a further study about the different type of risk and other factors that may have an influence on profit allocation, which is used to work out the profit allocation among players in coalition more objectively and reasonable. 


\section{References}

[1] K. Liu, Research on the Distribution of the Income on Real Estate, Shanxi University of Finance \& Economics, 2012.

[2] L. Liu, H. J. Tang, Z. J. Chen, Application of the Shapley Value in Game Analysis of Profit Distribution of Agile Virtual Enterprise, J. Industrial Engineering Journal. 6 (2006) 118-121.

[3] J. H. Dai, H. X. Xue, The Strategy of Profit Allocation among Partners in Dynamic Alliance Based on the Shapley Value, J. Chinese Journal of Management Science, 4 (2004) 33-36.

[4] H. H. Zhou, H. L. Bao, Research on the Profit Distribution among Technological Innovation Alliance Based on Improved Shapley Value, J. Science and Technology Management Research, 24 (2012) 109-112.

[5] Y. F. Shi, J. H. Gao, Innovation on Strategies of Profit Allocation in the Supply Chain Based on Shapley Value Method, J. Value Engineering, 25 (2011) 19-20.

[6] Mares M, Fuzzy Cooperative Games: Cooperation with Vague Expectations, New York Physica Verlag Press, 2001.

[7] Shapley L.S, A value for n-persons games, J. Annals of Mathematics Studies, 28 (1953) 307-318.

[8] W. Chen, Q. Zhang, Shapley Value for Fuzzy Cooperative Game, J. Journal of Management Sciences, 5 (2006) 50-55.

[9] X. H. Yu, Q. Zhang, Interval Shapley Value for Cooperative Games with Interval Payoffs, J. Fuzzy Systems and Mathematics, 5 (2008) 151-156.

[10] J. M. Wang, H. J. Du, Analysis of Cooperation Game with Random Payoff, J. Control and Decision, 1 (2010) 157-160.

[11] Judith Timmer, The Compromise Value for Cooperative Games with Random Payoffs, Math.Meth.oper.Res, 2006: 95-106.

[12] Suijs J, Borm P, De Waegenaere A, et al, Cooperative games with stochastic payoffs, J. European Journal of Operational Research, 113 (1) 1999 193-205.

[13] Y. J. Huang, J. Ying, A Study on the Coopetition in China's Commercial Banks: Significance and Strategies, J. Journal of Zhejiang University (Humanities and Social Sciences), 35 (7) 2005 60-68.

[14] Shapley L S, Shubik M, A method for evaluating the distribution of power in a committee system, J. American Political Science Review, 48 (03) 1954 787-792. 